\title{
Probing the hadronic nature of the gamma-ray emission associated with Westerlund 2
}

\author{
Enrique Mestre, ${ }^{1,2 \star}$ Emma de Oña Wilhelmi, ${ }^{1,2,3 \star}$ Diego F. Torres, ${ }^{1,2,4}$ Tim Lukas Holch, ${ }^{5}$ \\ Ullrich Schwanke, ${ }^{5}$ Felix Aharonian, ${ }^{6,7,8}$ Pablo Saz Parkinson, ${ }^{9,10}$ Ruizhi Yang ${ }^{\circledR 11,12,13}$ \\ and Roberta Zanin ${ }^{14}$ \\ ${ }^{1}$ Institute of Space Sciences (ICE/CSIC), Campus UAB, Carrer de Can Magrans s/n, E-08193 Barcelona, Spain \\ ${ }^{2}$ Institut d'Estudis Espacials de Catalunya (IEEC), E-08034 Barcelona, Spain \\ ${ }^{3}$ Deutsches Elektronen Synchrotron DESY, D-15738 Zeuthen, Germany \\ ${ }^{4}$ Institució Catalana de Recerca i Estudis Avançats (ICREA), E-08010 Barcelona, Spain \\ ${ }^{5}$ Humboldt University of Berlin, Newtonstr. 15, D-12489 Berlin, Germany \\ ${ }^{6}$ Dublin Institute for Advanced Studies, 31 Fitzwilliam Place, Dublin 2, Ireland \\ ${ }^{7}$ Max-Planck-Institut für Kernphysik, P.O. Box 103980, D-69029 Heidelberg, Germany \\ ${ }^{8}$ Gran Sasso Science Institute, 7 viale Francesco Crispi, I-67100 L'Aquila, Italy \\ ${ }^{9}$ Department of Physics and Laboratory for Space Research, The University of Hong Kong, Pokfulam Road, Hong Kong \\ ${ }^{10}$ Santa Cruz Institute for Particle Physics, University of California, Santa Cruz, CA 95064, USA \\ ${ }^{11}$ Department of Astronomy, School of Physical Sciences, University of Science and Technology of China, Hefei, Anhui 230026, China \\ ${ }^{12}$ CAS Key Labrotory for Research in Galaxies and Cosmology, University of Science and Technology of China, Hefei, Anhui 230026, China \\ ${ }^{13}$ School of Astronomy and Space Science, University of Science and Technology of China, Hefei, Anhui 230026, China \\ ${ }^{14}$ CTA Observatory GmbH, Via Piero Gobetti 93, I-40129 Bologna, Italy
}

Accepted 2021 May 18. Received 2021 May 18; in original form 2021 February 10

\begin{abstract}
Star-forming regions have been proposed as potential Galactic cosmic ray accelerators for decades. Cosmic ray acceleration can be probed through observations of gamma-rays produced in inelastic proton-proton collisions at $\mathrm{GeV}$ and $\mathrm{TeV}$ energies. In this paper, we analyse more than $11 \mathrm{yr}$ of Fermi-LAT data from the direction of Westerlund 2, one of the most massive and best-studied star-forming regions in our Galaxy. In particular, we investigate the characteristics of the bright pulsar PSR J10235746 that dominates the gamma-ray emission below a few $\mathrm{GeV}$ at the position of Westerlund 2 and the underlying extended source FGES J1023.3-5747. The analysis results in a clear identification of FGES J1023.3-5747 as the GeV counterpart of the TeV source HESS J1023-575, through its morphological and spectral properties. This identification provides new clues about the origin of the HESS J1023-575 gamma-ray emission, favouring a hadronic origin of the emission, powered by Westerlund 2, rather than a leptonic origin related to either the pulsar wind nebula associated with PSR J1023-5746 or the cluster itself. This result indirectly supports the hypothesis that star-forming regions can contribute to the cosmic ray sea observed in our Galaxy.
\end{abstract}

Key words: stars: winds, outflows - cosmic rays - open clusters and association: indivisdual: Westerlund 2 - gamma-rays: stars.

\section{INTRODUCTION}

The potential of massive star clusters to accelerate Galactic cosmic rays (GCRs) to very high energies (VHE, $E>100 \mathrm{GeV}$ ) has been recognized since the 1980s (Cesarsky \& Montmerle 1983; Binns et al. 2005; Bykov et al. 2020). Several hypotheses have been proposed for acceleration sites to very high energies in star-forming regions (SFRs), either in the vicinity of $\mathrm{OB}$ and WR stars or at the interaction of their fast winds with supernova ( $\mathrm{SN}$ ) shocks, or in so-called super-bubbles (see e.g. Bykov et al. 2020; Gabici et al. 2019 and references therein). The presence of cosmic rays (CRs) can be inferred by means of gamma-ray observations (above a few hundreds of $\mathrm{MeV}$ ) by looking at the byproduct of inelastic proton-proton collisions. The spectral energy distribution (SED)

^E-mail: mestre@ice.csic.es (EM); wilhelmi@ice.csic.es (EdOW) from hadronic-originated gamma-ray sources is characterized by a sharp rise in the $\sim 70-200 \mathrm{MeV}$ range (resulting from the neutral pion threshold production energy), followed by a hard emission up to the maximum energy. Competing gamma-ray processes related to leptonic emission should have different imprints on the spectral shape, showing a (in many occasions broad) peak in the hundreds of $\mathrm{GeV}$ range. Therefore, sampling the spectrum from a few hundreds of $\mathrm{MeV}$ to tens of $\mathrm{TeV}$ should result in a strong indication of the origin of the observed radiation. Several SFRs have been identified as likely GCR accelerators in the $\mathrm{GeV}$ and $\mathrm{TeV}$ regime (Ackermann et al. 2011a; H. E. S. S. Collaboration 2011; Abramowski et al. 2012; H. E. S. S. Collaboration 2015; Yang, de Oña Wilhelmi \& Aharonian 2018; Aharonian, Yang \& de Oña Wilhelmi 2019; Saha et al. 2020; Yang \& Wang 2020; Sun, Yang \& Wang 2020). However, a firm identification remains elusive, given the large extension of the sources and/or the presence of some other efficient accelerators in the region, such as pulsars and pulsar wind nebulae (PWNe). 
One of the most massive and well-studied SFRs in our Galaxy is Westerlund 2 . The cluster itself presents a $\sim 0.2^{\circ}$ wind-blown bubble observed in infrared (IR) by Spitzer and in radio continuum by Australia Telescope Compact Array (ATCA, Whiteoak \& Uchida 1997; Whitney et al. 2004). This bubble is coincident with a prominent feature at radio wavelengths, known as the blister, and with an extended $\mathrm{TeV}$ source $\left(\sim 0.2^{\circ}\right)$ known as HESS J1023-575 (Aharonian et al. 2007; H. E. S. S. Collaboration 2011). In the GeV regime, the young and energetic pulsar PSR J1023-5746 lies 8 arcmin away from the cluster, and its PWN was initially suggested as possible counterpart of the TeV source (Saz Parkinson et al. 2010; Ackermann et al. 2011b) (see Table 2 for the physical parameters of the pulsar, obtained from Saz Parkinson et al. 2010). Besides the PWN scenario, several accelerators have been proposed to power the $\mathrm{TeV}$ emission: Westerlund 2 contains an extraordinary ensemble of hot and massive OB stars (Rauw et al. 2004) for which a total energy release for the collective winds was estimated to be $\sim 5.7 \times 10^{37} \mathrm{erg}$ $\mathrm{s}^{-1}$. Westerlund 2 also hosts one of the most massive binary systems, composed of two WN6ha stars (Rauw et al. 2004), dubbed WR 20a. Their orbital period is $\sim 3.6 \mathrm{~d}$ (Bonanos et al. 2004; Rauw et al. 2004) and the estimated kinetic energy loss rate is $\sim 10^{37} \mathrm{erg} \mathrm{s}^{-1}$. Several massive molecular clouds were found within the surrounding of Westerlund 2, overlapping with the $\mathrm{GeV}$ and $\mathrm{TeV}$ gamma-ray sources (see fig. 5 in H. E. S. S. Collaboration 2011).

Since the discovery of the Westerlund 2 cluster, there have been several attempts to determine accurately its distance, estimated between $2 \mathrm{kpc}$ and $8 \mathrm{kpc}$ (Salpeter (Salpeter 1955; Moffat, Shara \& Potter 1991; Piatti, Bica \& Claria 1997; Carraro \& Munari 2004; Rauw et al. 2005; Uzpen et al. 2005; Ascenso et al. 2007; Dame 2007; Rauw et al. 2007; Tsujimoto et al. 2007; Furukawa et al. 2009; Vargas Álvarez et al. 2013). In the following, we adopt a value of $5 \mathrm{kpc}$, roughly in the mid-range of the recent optical photometric work (Vargas Álvarez et al. 2013; Drew et al. 2018) and at the bottom end of the interstellar medium (ISM) estimates (Dame 2007; Furukawa et al. 2014).

The Large Area Telescope (LAT, Atwood et al. 2009) onboard the Fermi Gamma-ray Space Telescope, launched in 2008 June, surveys the gamma-ray sky in the $20 \mathrm{MeV}$ to greater than $300-\mathrm{GeV}$ energy range. The LAT data recorded during the last decade have resulted in different catalogues of gamma-ray sources (Ballet et al. 2020). For instance, the 4FGL catalogue (Abdollahi et al. 2020) covers the data recorded during the first $8 \mathrm{yr}$ of observations. On the other hand, the extended sources are listed in the Fermi Galactic Extended Source (FGES) Catalog (Ackermann et al. 2017a). In this paper, we analysed the gamma-ray emission towards FGES J1023.3-5747 (Ackermann et al. 2017b) and the pulsar PSR J1023-5746 taking advantage of more than $11 \mathrm{yr}$ of Fermi-LAT data and the most recent gammaray source catalogue released by the Fermi-LAT collaboration. The paper is structured as follows. In Sections 2 and 3, we describe the collected data and present the results of the analysis. In Section 4, we discuss the possible leptonic (Section 4.1) or hadronic (Section 4.2) origin of the FGES J1023.3-5747 gamma-ray extended emission. Finally, in Section 5, we summarize the conclusions reached.

\section{DATA ANALYSIS}

To investigate the characteristics of the gamma-ray emission from FGES J1023.3-5747 and PSR J1023-5746, we used Fermi-LAT (P8R3, Atwood et al. 2013; Bruel et al. 2018) data spanning from 2008 August 4 to 2019 April 24 (or 239557417-577782027 seconds in Fermi Mission Elapsed Time) in the energy range from $200 \mathrm{MeV}$ to $500 \mathrm{GeV}$. We retrieved the data from a region of interest (ROI) defined by a radius of $20^{\circ}$ around the position of PSR J1023-5746 $\left(\mathrm{RA}=155.76^{\circ}, \mathrm{DEC}=-57.77^{\circ}\right.$, Kerr et al. 2015). We analysed only the dubbed SOURCE class events with a maximum zenith angle of $90^{\circ}$ to eliminate Earth limb events. The events were selected with a minimum energy of $200 \mathrm{MeV}$ to avoid events poorly reconstructed due to the large angular resolution and the large crowding of sources in the region. The analysis of the source spectrum below $200 \mathrm{MeV}$ is crucial to characterize hadronic-originated gamma-rays sources, since neutral pion decay spectrum rises steeply below this energy, a feature often referred to as the pion-decay bump. However, this particular source is not a promising candidate for this analysis: the moderate flux of the source and hard spectrum limits the number of photons in the very low-energy regime; the extended morphology complicates the analysis with respect to the point-like case; the presence of several sources in the region may contaminate further the spectrum especially at low energies, preventing an accurate characterization (i.e. overlapping with the PSR J1023-5746 emission). In this work, we focus on studying the general $\mathrm{GeV}$ to $\mathrm{TeV}$ spectral shape.

To obtain the spectrum and morphology of FGES J1023.3-5747, we first had to disentangle its diffuse gamma-ray emission from the radiation coming from the pulsar PSR J1023-5746 and other nearby sources. For that purpose, we need to derive first the SED of all the sources in the field of view. Therefore, a comprehensive model describing the gamma-ray sources in the ROI is needed. In order to build this model, we included all the LAT sources listed in the Fermi-LAT Fourth Source Catalog (4FGL, Abdollahi et al. 2020) in a radius of $20^{\circ}$ around the position of FGES J1023.3-5747. On the other hand, the LAT data contain a significant contribution from Galactic and extra-galactic diffuse gamma-rays, which is described with the latest version available of the Galactic (gll_iem_v07) and isotropic (iso_P8R3_SOURCE_V2_v1) diffuse emission models. The model's free parameters correspond to the ones of the sources within $3^{\circ}$ around the position of PSR J1023-5746. Beyond this radius, the normalization of all the sources with test statistic greater than 10 (TS $>10)$ is also free. The Test Statistic is defined as TS $=2 \log \left(\mathrm{L} / \mathrm{L}_{0}\right)$, where $L$ is the maximum value of the likelihood function over the ROI including the source in the model and $L_{0}$ is the same without the source (Mattox et al. 1996). Hence, the detection significance of a source is usually approximated by the square root of the TS.

The analysis of the LAT data described above was performed by means of the FERMIPY PYTHON package (version 0.18.0), based on the FERMI SCIENCE TOOLS (Wood et al. 2017). The data were binned in eight energy bins per decade and spatial bins of $0.1^{\circ}$ size to perform the analysis. The response of the LAT instrument is evaluated with the Instrument Response Functions (IRF, version P8R3_SOURCE_V2). The energy dispersion correction was applied to the sources in our model, except for the isotropic diffuse emission model.

The current version of LAT data (i.e. PASS 8) classifies the events into quartiles based on the varying quality of the direction reconstruction (PSF events types). In our analysis, we took into account the four point spread function (PSF) event types available (dubbed PSF0, PSF1, PSF2, and PSF3) and applied the appropriate IRF and isotropic background models according to the quality of the reconstructed event directions. Doing so, we are preventing the loss of possibly useful information in the analysis by means of the separate treatment of high-quality events and poorly localized ones. The analysis is performed by means of a joint likelihood fitting process.

In order to discriminate the diffuse gamma-ray emission of FGES J1023.3-5747 from the emission of the nearby pulsar, we first 
performed a timing analysis on the PSR J1023-5746 data to gate the pulsed emission by means of the pulsar analysis package TEMPO2 (Hobbs, Edwards \& Manchester 2006). To obtain the phase curve of PSR J1023-5746, we assigned the corresponding phases to the gamma-ray events localized in a region of $0.6^{\circ}$ of radius around the pulsar position $\left(\mathrm{RA}=155.76^{\circ}, \mathrm{DEC}=-57.77^{\circ}\right)$. Then, we computed the phases of the events using the updated ephemeris of PSR J1023-5746 at epoch 55635 MJD. The new ephemeris that allows us to enlarge the data set to more than $11 \mathrm{yr}$ was provided by the Fermi-LAT collaboration. Finally, we applied the Bayesian Blocks method (Scargle et al. 2013) to the PSR J1023-5746 light curve and obtained different components, defined as ON, OFF, and Bridge emission. We computed the contribution to the phase curve of each of the sources in the model with the Fermi tool gtsrcprob, which assigns to every event the probability of belonging to each source of the model.

The SEDs of the sources were obtained for 12 energy bins (spanning from $200 \mathrm{MeV}$ to $500 \mathrm{GeV}$ ) using the whole ROI, with the FGES J1023.3-5747 and PSR J1023-5746 spectra characterized by power law and exponential cutoff power-law models, respectively. The sources of the SEDs are computed for the different components of the phase curve, cutting the events in phase. We tested the spectral analysis consistency studying the systematic uncertainties on the SEDs, mainly due to the LAT effective area $\left(\mathrm{A}_{\text {eff }}\right)$ and the Galactic diffuse emission model. The systematic uncertainties due to the LAT effective area are computed with the bracketing $\mathrm{A}_{\text {eff }}$ method, ${ }^{1}$ and the ones due to the diffuse Galactic model were tested by artificially changing the normalization of the same by \pm 6 per cent with respect to the best-fitting value (Ajello et al. 2011; Li et al. 2018).

On the other hand, the size of FGES J1023.3-5747 was analysed with the FERMIPY extension method, based on a likelihood ratio test with respect to the point-source hypothesis. We tested both 2D symmetric Gaussian and 2D radial disc models for the morphology of FGES J1023.3-5747. The best-fitting extension in each case is computed performing a likelihood profile scan over the source width (68 per cent containment) and maximizing the model likelihood.

Finally, we imposed different additional cuts of energy on the data described above (at $700 \mathrm{MeV}, 3 \mathrm{GeV}, 10 \mathrm{GeV}, 40 \mathrm{GeV}, 70 \mathrm{GeV}$, and $135 \mathrm{GeV}$ ) and studied the morphological characteristics of FGES J1023.3-5747 re-analysing the data in differential energy bins (see Fig. A1).

\section{RESULTS}

The analysis of the pulsed emission from PSR J1023-5746 with the Bayesian Blocks method showed two peaks, spanning from 0 to 0.2 , and from 0.43 to 0.58 in phase, with a Bridge emission in between. The peaks are centred at $\approx 0.09$ and $\approx 0.54$ in phase, respectively, and present a similar width $(\sigma \approx 0.02$ in phase if fitted with a Gaussian profile). The pulsed emission was analysed in three intervals of energy: from $200 \mathrm{MeV}$ to $700 \mathrm{MeV}$, from $700 \mathrm{MeV}$ to $3 \mathrm{GeV}$, and above $3 \mathrm{GeV}$. No significant shift was observed between the peaks at different energies. Also, the relation between the height of the peaks and between the number of events in each peak $(\mathrm{P} 1 / \mathrm{P} 2 \approx$ 0.8 ) does not differ significantly for the different energy bins. The second peak is the dominant one in the three intervals of energy. The number of events in the Bridge component (for the energy bins

${ }^{1}$ https://fermi.gsfc.nasa.gov/ssc/data/analysis/scitools/Aeff_Systematics.h $\mathrm{tml}$

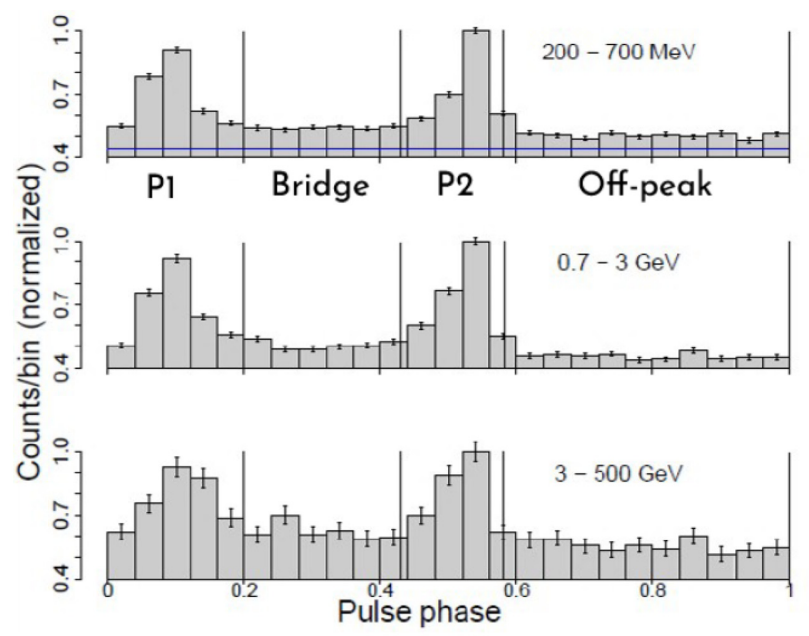

Figure 1. Phase curve of PSR J1023.1-5745 obtained at different energies in a region of $0.6^{\circ}$ around the pulsar position (normalized by the height of P2) with the different components of the phase curve noted. The horizontal blue line corresponds to the expected contribution in the phase curve of background sources (i.e. all the sources in the field of view except for FGES J1023.3-5747 and PSR J1023-5746).

mentioned) is $\sim 10$ per cent larger than the one expected from the offpeak statistics. The three pulse profiles in the different energy bins are shown in Fig. 1 (counts per bin, for each phase). The Galactic diffuse emission is dominant both in the Bridge and off-peak regions. Hence, a similar level (in counts per bin) is observed in Fig. 1 for both components. The same contribution from FGES J1023.3-5747 is expected in each bin of the phase curve (for a given energy interval), since the extended source emission should not vary in synchrony with the pulsed one.

PSR J1023-5746 (described as a point-like source with an exponential cutoff power-law spectrum) is located at $\mathrm{RA}=155.772^{\circ} \pm$ $0.005^{\circ}$ and DEC $=-57.764^{\circ} \pm 0.005^{\circ}$ (see Table A1). The source is well detected in the on-peak and Bridge regions with $\sqrt{T S} \gtrsim 80$ in both intervals. The results of the spectral analysis of PSR J10235746, performed both in the on-peak and Bridge intervals, are summarized in Table 1 (see Fig. 2 left-hand panel). Note that the SED is characterized by an exponential cutoff located at an energy of $\sim 3 \mathrm{GeV}$. Also, the cutoff energy obtained in the on-peak region is in agreement with the best-fitting one for the Bridge component, while the spectral indices in both analyses are compatible within the uncertainties (at 95 per cent confidence level (CL)). In addition, the positions fitted for PSRJ1023-5746, both in the on-peak and Bridge regions, are in agreement at 95 percent CL. The point-like source, however, is not detected if selecting only off-peak events. In this region, only upper limits for the flux of PSR J1023-5746 can be derived (see the red arrows in Fig. 2 left-hand panel). This argues in favour of a reduced contribution of the pulsar emission in the defined 'OFF' region in comparison to the 'Bridge'. Admittedly, however, a close flux level between both such regions complicates this distinction.

To investigate the emission associated with the extended source FGES J1023.3-5747, we analyse the off-peak interval (from 0.58 to 1 in phase) to minimize the contamination from PSR J1023-5746. The analysis results in a significant detection of FGES J1023.3-5747 $(\sqrt{\mathrm{TS}} \approx 14)$, with the source located at $\mathrm{RA}=155.93^{\circ} \pm 0.03^{\circ}$ and $\mathrm{DEC}=-57.79^{\circ} \pm 0.03^{\circ}$ (see Table A1). The parameters of the bestfitting power-law spectrum for FGES J1023.3-5747 are summarized 
Table 1. Best-fitting models for PSR J1023-5746 and FGES J1023.3-5747. The units of $N_{0}$ are $\mathrm{cm}^{-2} \mathrm{~s}^{-1} \mathrm{MeV}^{-1}$ for PSR J1023-5746 and $\mathrm{cm}^{-2} \mathrm{~s}^{-1} \mathrm{MeV}^{-1} \mathrm{sr}^{-1}$ in the case of FGES J1023.3-5747.

\begin{tabular}{lccc}
\hline Parameter & PSR J1023-5746 (on-peak) & PSR J1023-5746 (Bridge $)$ & FGES J1023.3-5747 (off-peak) \\
\hline$N_{0}$ & $\left(2.51 \pm 0.18_{\text {stat }} \pm 0.24_{\text {sys }}\right) \times 10^{-11}$ & $\left(6.57 \pm 1.22_{\text {stat }} \pm 2.44_{\text {sys }}\right) \times 10^{-12}$ & $\left(1.02 \pm 0.14_{\text {stat }} \pm 0.16_{\text {sys }}\right) \times 10^{-14}$ \\
$\Gamma$ & $1.74 \pm 0.05_{\text {stat }} \pm 0.16_{\text {sys }}$ & $1.61 \pm 0.17_{\text {stat }} \pm 0.56_{\text {sys }}$ & $2.05 \pm 0.06_{\text {stat }} \pm 0.33_{\text {sys }}$ \\
$E_{0}[\mathrm{GeV}]$ & 1.95 & 1.95 & 17 \\
$E_{\text {cutoff }}[\mathrm{GeV}]$ & $2.74 \pm 0.24_{\text {stat }} \pm 0.39_{\text {sys }}$ & $2.7 \pm 0.57_{\text {stat }} \pm 1.2_{\text {sys }}$ & - \\
$\sigma[\mathrm{deg}]$ & - & - & $0.16 \pm 0.02$ \\
Parameter & FGES J1023.3-5747 $(E>10 \mathrm{GeV})$ & FGES J1023.3-5747 (on-peak) & FGES J1023.3-5747 (Bridge) \\
$N_{0}$ & $\left(1.09 \pm 0.16_{\text {stat }} \pm 0.04_{\text {sys }}\right) \times 10^{-14}$ & $\left(1.03 \pm 0.21_{\text {stat }} \pm 0.10_{\text {sys }}\right) \times 10^{-14}$ & $\left(9.83 \pm 2.0_{\text {stat }} \pm 0.5_{\text {sys }}\right) \times 10^{-15}$ \\
$\Gamma$ & $2.07 \pm 0.15_{\text {stat }} \pm 0.02_{\text {sys }}$ & $\left(2.01 \pm 0.16_{\text {stat }} \pm 0.26_{\text {sys }}\right)$ & $\left(2.11 \pm 0.14_{\text {stat }} \pm 0.22_{\text {sys }}\right)$ \\
$E_{0}[\mathrm{GeV}]$ & 17 & 19.6 & - \\
$E_{\text {cutoff }}[\mathrm{GeV}]$ & - & - & $0.16 \pm 0.04$ \\
$\sigma[\mathrm{deg}]$ & $0.15 \pm 0.02$ & & $0.16 \pm 0.03$ \\
\hline
\end{tabular}
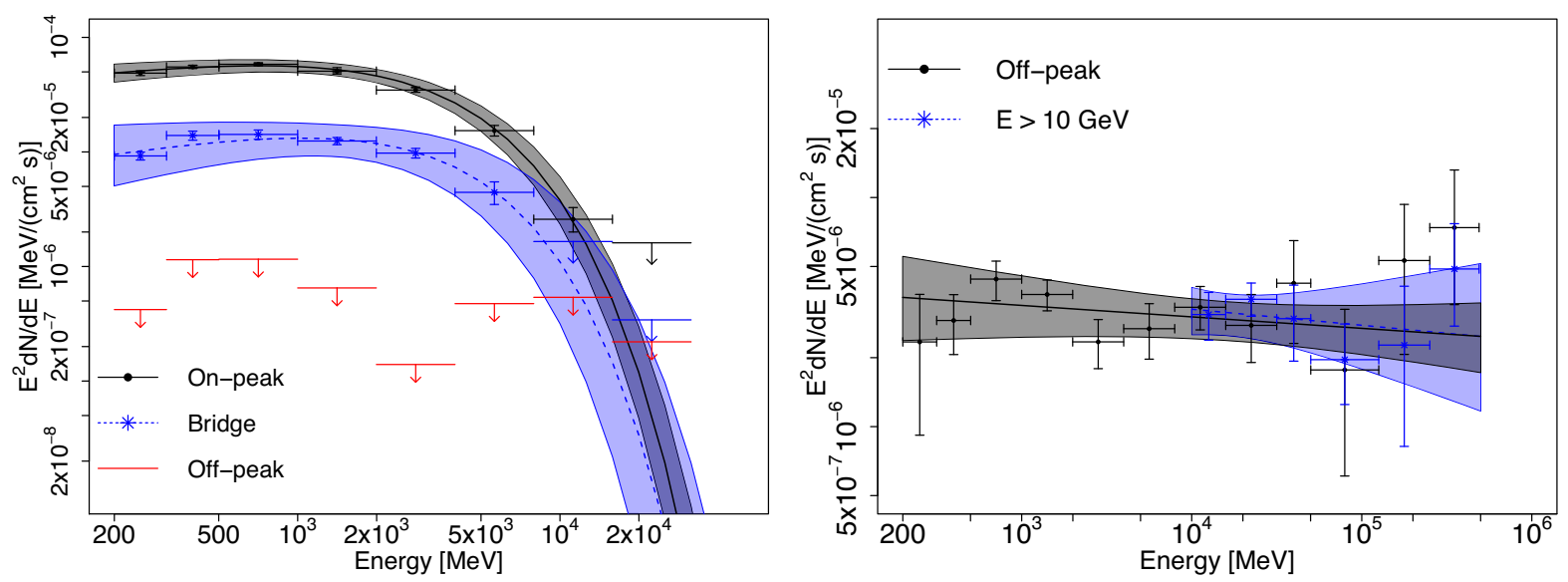

Figure 2. On the left (a): Spectral energy distribution (with only statistical errors) of PSR J1023-5746 derived from the on-peak (in black, with dot markers), the Bridge (in blue, with star markers), and the off-peak regions (red upper limits). On the right (b): Spectral energy distribution (with only statistical errors) derived from FGES J1023.3-5747 after gating the pulsar emission (in black) and analysing only the gamma-ray emission above $10 \mathrm{GeV}$ (without cutting in phase, in blue). The shaded area marks the $1 \sigma$ error on the fitted spectral model.

in Table 1 (see Fig. 2 right-hand panel). The source spatial component is best described by a symmetric 2D Gaussian model, and the fit of the extension resulted in a 68 per cent containment radius of $r_{68}=0.24^{\circ}$ $\pm 0.03^{\circ}$ (i.e. an intrinsic size of $\sigma=0.16 \pm 0.02$, see Lande et al. 2012), with a $0.29^{\circ}$ upper limit for the 95 per cent containment radius. We also tested a symmetric 2D disc model, which, however, does not improve the log-likelihood with respect to the best-fitting Gaussian model, obtaining a 68 per cent containment radius compatible with the extension above (i.e. $0.25^{\circ} \pm 0.02^{\circ}$ ). Also, the log-likelihood of the best-fitting Gaussian model with an extension fixed to the value measured by H.E.S.S. $\left(\sigma=0.18^{\circ}\right.$, H. E. S. S. Collaboration 2011) is not significantly smaller than the one corresponding to the best-fitting extension described above $(\Delta \mathrm{TS} \approx 1.6$, as expected, given the similar extension of the $\mathrm{GeV}$ and $\mathrm{TeV}$ excesses). The extension and position of FGES J1023.3-5747 were also computed for the on-peak and Bridge components, where an extended source model was fitted simultaneously to the PSR J1023-5746 emission. No significant difference with respect to the results in the off-peak region was observed. The 'OFF' region of PSR J1023-5746 has been analysed with LAT data in previous works. In the second catalogue of LAT gamma-ray pulsars (2PC, see Abdo et al. 2013), an unidentified source was reported in the off-pulse region, defined from 0.76 to 0.02 in phase. The analysis of this source showed some indication of spatial extension $\left(\mathrm{TS}_{\mathrm{ext}}=30\right)$. Interestingly, the integrated flux for such source, computed from $100 \mathrm{MeV}$ to $316 \mathrm{GeV}$ of energy, i.e. $1.79 \times 10^{-8} \mathrm{~cm}^{-2} \mathrm{~s}^{-1}$ (with large uncertainty) is compatible with the one derived (in this work) for FGES J1023.3-5747 in the same regime (of energy), i.e. $(1.97 \pm 0,49) \times 10^{-8} \mathrm{~cm}^{-2} \mathrm{~s}^{-1}$ (with only statistical errors). Similarly, the off-peak region was analysed in Ackermann et al. 2011b, with 16 months of LAT data. In this case, the off-peak emission was detected only at energies above 10 $\mathrm{GeV}$. Despite the off-pulse region (from 0.85 to 1.13 in phase, in the cited paper) differs significantly from the definition in this work, and the small amount of data used compared to the reanalysis we present, the integral flux of the source reported (from $10 \mathrm{GeV}$ to 100 $\mathrm{GeV}$ of energy), i.e. $(4.6 \pm 2.2) \times 10^{-10} \mathrm{~cm}^{-2} \mathrm{~s}^{-1}$ is also in well agreement with the flux derived for FGES J1023.3-5747 in the same interval $(2.62 \pm 0.37) \times 10^{-10} \mathrm{~cm}^{-2} \mathrm{~s}^{-1}$. A more recent reanalysis of the detected off-pulse emission with 45 months of LAT data (see Acero et al. 2013) reported a similar integrated flux above $10 \mathrm{GeV}$ of energy.

The position of the sources, fitted for the different components of the phase curve, implies a separation between PSR J1023-5746 and the centroid of FGES J1023.3-5747 of 5.3 \pm 1.1 arcmin, with the pulsar position well within the extended source (given the size measured for the same, see Fig. 3). In addition, the best-fitting position of FGES J1023.3-5747 (in the off-peak region) is in good agreement with the one estimated by H.E.S.S for HESS J1023-575 


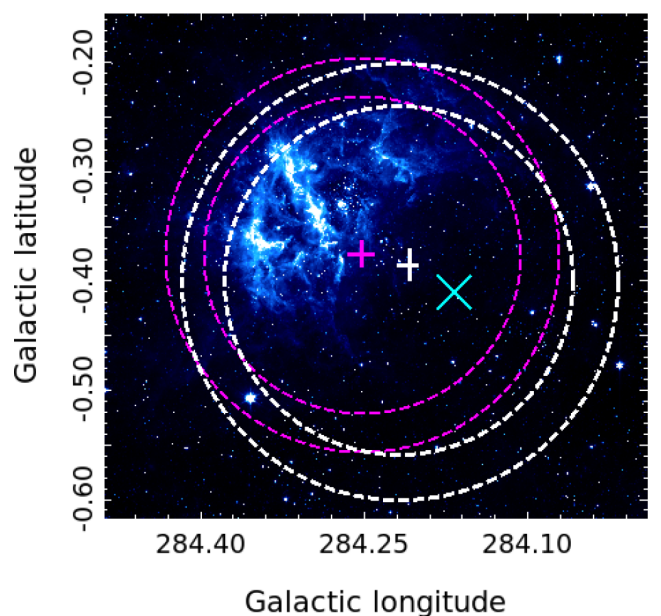

Figure 3. Spitzer/IRAC GLIMPSE Mosaic obtained from the GLIMPSE survey archival data. The magenta dashed lines correspond to the $1 \sigma$ interval for the best-fitting extension of FGES J1023.3-5747, with the central cross marking the best-fitting position (with the $1 \sigma$ error). The white dashed lines and cross correspond, similarly, to the morphological characteristics of the H.E.S.S. source HESS J1023-575, as described in H. E. S. S. Collaboration 2011. The cyan cross corresponds to the position of PSR J1023-5746.

(i.e. $\mathrm{RA}=155.85^{\circ}$ and $\mathrm{DEC}=-57.79^{\circ}, \mathrm{H}$. E. S. S. Collaboration 2011), with a separation between the centroids of the sources of $3.3 \pm 1.4$ arcmin (account statistical errors only).

To further investigate the spectrum and morphology of the high energy part of FGES J1023.3-5747, we analysed the data set above $10 \mathrm{GeV}$ (see Table 1 and Fig. 2 right-hand panel), where additional cuts in phase are not necessary due to the exponential decrease of the pulsed emission above $\sim 3 \mathrm{GeV}$ (of energy). We included the emission of PSR J1023-5746 (modelled as a point-like source with the spectrum shown in Table 1) in this analysis, detected with low significance at energies above $10 \mathrm{GeV}(\sqrt{\mathrm{TS}} \approx 3)$. The extension fitted in this case for FGES J1023.3-5747 was $r_{68}=0.23^{\circ}+0.03^{\circ}-$ $0.02^{\circ}$ (for a $2 \mathrm{D}$ Gaussian model). Note that both the position of the extended source and the best-fitting power-law spectral model are in good agreement with the ones obtained in the off-peak region (at 95 per cent CL, see Fig. 2 right-hand panel and Table A1). Also, the spectral index, obtained both in the off-peak region and at energies above $10 \mathrm{GeV}$, is compatible with $\Gamma=2$, within the uncertainties (at 95 per cent CL).

To conclude, we studied the morphology of FGES J1023.3-5747 (in the off-peak component) in six energy bins (with breakpoints in $200 \mathrm{MeV}, 700 \mathrm{MeV}, 3 \mathrm{GeV}, 10 \mathrm{GeV}, 40 \mathrm{GeV}, 70 \mathrm{GeV}$, and $500 \mathrm{GeV}$ ). For this purpose, we fitted the extension of FGES J1023.3-5747 (measured for a Gaussian profile, i.e. 68 per cent containment radius) as a function of the energy (see Fig. A1). Then, we tested an energydependent shrinking model of the form $\sigma \propto E^{-\alpha}$, but no significant variation of FGES J1023.3-5747 extension was observed. The flux fitted for FGES J1023.3-5747 in each bin of energy is compatible with the one expected from the spectrum fitted in the full range of energy (from $200 \mathrm{MeV}$ to $500 \mathrm{GeV}$ ), and the spectral index of the best-fitting model is compatible with $\Gamma=2$ (at $3 \sigma$ ) for each bin of energy. However, the angular resolution of Fermi-LAT $\left(\sim 0.8^{\circ}\right.$ at $1 \mathrm{GeV}$, see Abdollahi et al. 2020) limits the fit of the FGES J1023.35747 extension for energies below few GeVs. Note that, since the Fermi-LAT PSF is $\sim 0.2^{\circ}$ at $3 \mathrm{GeV}$ ( 68 per cent containment radius), an imperfect diffuse modelling can bias the extension measured at lower energies. The large-scale diffuse emission, dominant at low energies $(\sim 500 \mathrm{MeV})$, limits the extension measurements performed in this regime, as seen in Fig. A1 (note the upper limit in the lowest energy bin). The contamination from the same, however, is decreasingly relevant at higher energies.

\section{DISCUSSION}

The gamma-ray emission from the bright GeV pulsar PSR J10235746 dominates the emission below a few $\mathrm{GeV}$. Within the current statistics, the light curve remains similar in different energy ranges, contrary to other pulsars such as Crab or Vela. It is well described by two narrow peaks and a Bridge region between them. The spectrum obtained from this region is very similar to the one from the peaks, with an exponential cutoff at $\sim 3 \mathrm{GeV}$, indicating a common location of the radiation zone, most likely within the pulsar magnetosphere or its vicinity.

The analysis of the gamma-ray emission, gating off the On- and Bridge-regions, combined with the large data set, allows the detailed investigation of the spectral shape and morphology of the extended underlying source FGES J1023.3-5747. The measured size of the $\mathrm{GeV}$ source is in good agreement with the one measured by H.E.S.S. for HESS J1023-575 (see Fig. 3). The spectrum obtained from the $\sim 0.2^{\circ}$ region is hard $(\Gamma=2)$ and connects smoothly the emission observed with LAT with the one in the TeV regime (see Fig. 4), indicating a clear identification of FGES J1023.3-5747 as the GeV counterpart of HESS J1023-575. The spectral results agree with the ones reported in Ackermann et al. (2017b) but are in tension with the ones obtained by Yang et al. (2018). For the latter, the ephemeris used was valid only for a reduced period in comparison to the temporal span of the data analysed, resulting most likely in a contamination from the pulsar that affected the spectrum of the extended source. For this work, in turn, we have used the most updated ephemeris for the pulsar, valid for the entire data set analysed.

The new characterization of the emission, thanks to the larger data set and better source discrimination, provides new clues to establish the origin of the gamma-ray emission. Different scenarios are discussed in the following, in the context of the new morphology and spectral features found.

\subsection{PWN scenario}

The discovery of the energetic pulsar PSR J1023-5746 (with spindown energy $\dot{\mathrm{E}}=10^{37} \mathrm{erg} \mathrm{s}^{-1}$ ), together with the proven efficiency of PWNe to produce TeV gamma-rays (H. E. S. S. Collaboration 2018), triggered the interpretation of HESS J1023-575 as a PWN energized by PSR J1023-5746. The association of the stellar cluster with the birth site of PSR J1023-5746 ( $\tau_{\mathrm{c}}=4.6 \mathrm{ky}$, Saz Parkinson et al. 2010) is not straightforward, implying an unrealistic transverse velocity of the pulsar (Ackermann et al. 2011b) if the same is located at the distance the cluster is believed to be $\sim 6 \mathrm{kpc}$ (Dame 2007). Several time-dependent modellings of a Pulsar/PWN scenario have been proposed to connect HESS J1023-575 with PSR J1023-5746 (Ackermann et al. 2011b; H. E. S. S. Collaboration 2018). In the reanalysis presented here, we found a noticeable morphological (see Fig. 3) and spectral (see Fig. 4) overlap between the extended GeV source FGES J1023.3-5747 and the TeV source HESS J1023-575, which provides strong constraints on the PWN interpretation. In the same, the gamma-ray spectrum is expected to show a peak at energies just below the cutoff energy in the electron spectrum in the $\mathrm{TeV}$ regime. The GeV gamma-ray spectrum due to the interactions of electrons with magnetic and radiation fields is effectively uncooled, up to the cutoff energy, since IC and synchrotron loss times are much longer 


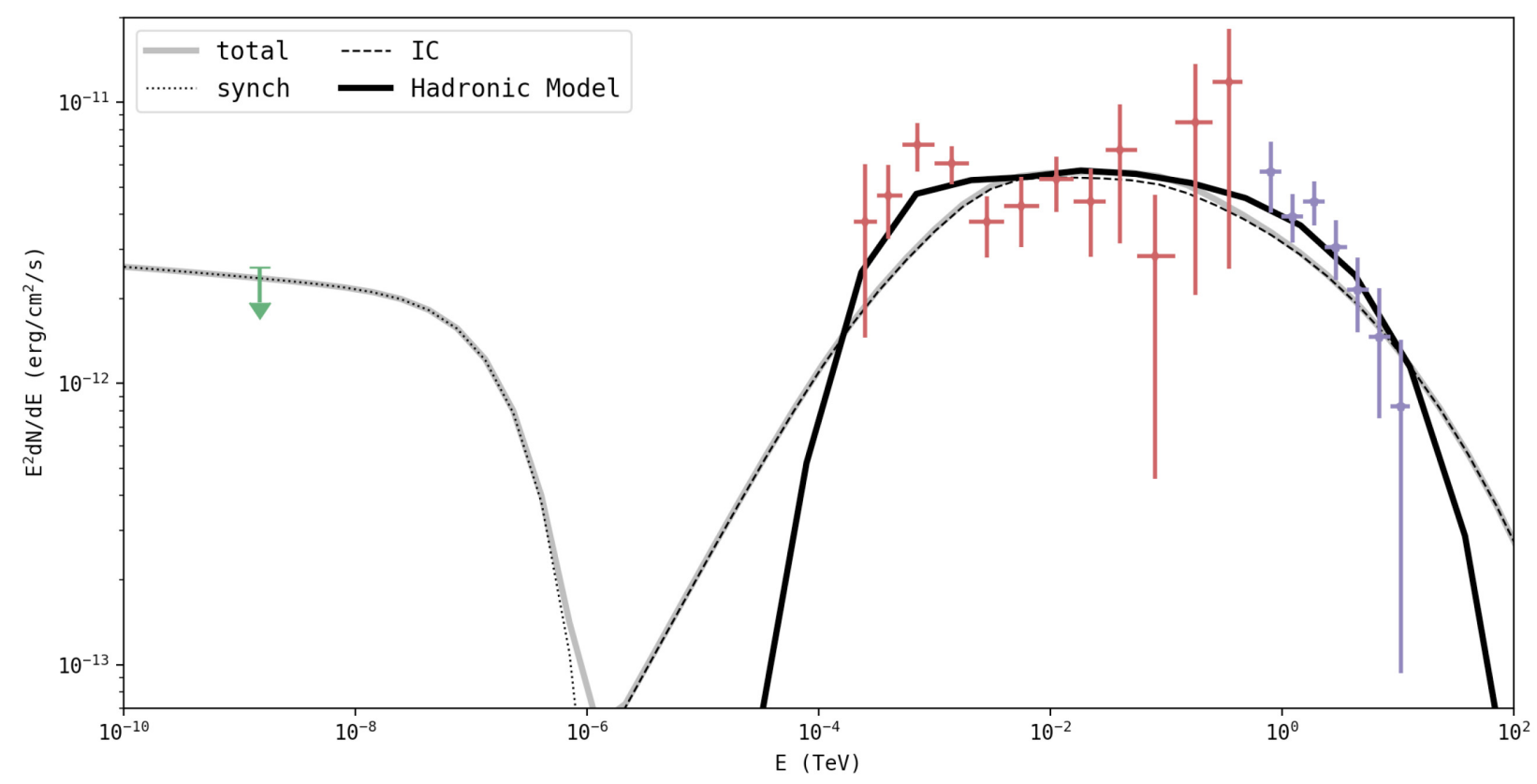

Figure 4. Best-fitting models for FGES J1023.3-5747 spectrum in the pion decay (solid line) and PWN (dashed line) hypotheses. The red points correspond to the Fermi-LAT data analysis (with only statistical errors) and the purple ones to H.E.S.S. (H. E. S. S. Collaboration 2011). The upper limit in the X-ray domain is obtained from Fujita et al. (2009).

in a typical PWN environment. The IC cooling time corresponding to the photon fields in the region where the pulsar is located (dust IR photons, stellar photons, and CMB, see Table 2 where the density of the dust/stellar, $\omega_{\mathrm{FIR} / *}$ at temperature $\mathrm{T}_{\mathrm{FIR} / *}$ is given) is $>10^{4} \mathrm{yr}$, whereas a magnetic field of $>400 \mu \mathrm{G}$ should be considered to cool efficiently a few $\mathrm{GeV}$ electrons $\left(\tau_{\mathrm{syn}, \mathrm{yr}} \simeq 1.3 \times 10^{7} B_{\mu \mathrm{G}}^{-2} E_{\mathrm{e}, \mathrm{TeV}}^{-1}\right)$. The uncooled $\Gamma_{\gamma} \sim 2$ spectrum extends from $\sim 200 \mathrm{MeV}$ to a few hundred $\mathrm{GeV}$, where it meets the one measured by H.E.S.S. (see Fig. 4). This photon spectrum would correspond to an electron spectrum of index $\alpha \approx 2 \Gamma_{\gamma}-1=3$ (in the Thompson regime, Blumenthal \& Gould 1970). Such a spectral index is difficult to reconcile with acceleration theories and typical injection spectra in PWNe. To further investigate this, we model the data using a PWN radiative scenario, in which the particle evolution and radiation are evolving in time according to the approach taken in Torres et al. (2014). The time-dependent gammaray spectrum is obtained using the GAMERA software (Hahn 2015). Likewise, we used a broken power-law injection spectrum with similar shape to those found for young PWNe (see Table 2 for details and table 2 in Torres et al. 2014). The time-dependent modelling used does not account for morphological changes (i.e. assumes energyindependent morphology). The best model representing the data is shown in Fig. 4, integrating in time up to the estimated pulsar age $\left(\tau_{\mathrm{c}} \sim 4.6 \mathrm{kyr}\right)$. The upper limit on the X-ray emission was obtained by Fujita et al. (2009) using Suzaku observations (in a $17^{\prime} .8 \times 17^{\prime} .8$ field). The disagreement between the LAT data and the model is evident, especially at the lower energies, and one should then consider a more complex modelling, with more than one electron population, to obtain a good representation of the data. According to Manolakou, Horns \& Kirk (2007), the stellar average density associated with Westerlund 2 could be as high as $500 \mathrm{eV} / \mathrm{cm}^{3}$ at a temperature of $3 \times 10^{4} \mathrm{~K}$, well beyond the nominal $2 \mathrm{eV} / \mathrm{cm}^{3}$ density used in the model described in Table 2. However, we found no energy density in the cited range for which a better fit than that depicted in Fig. 4 is derived (see Fig. A2). A second possibility to flatten the spectrum involves the contribution of different stellar photon fields, which would be indeed expected from a region like Westerlund 2 (Guarnieri et al. 1995; Vargas Álvarez et al. 2013). However, the morphology of such a source, peaking at the regions of high stellar radiation density, would differ from the one observed, rendering this possibility unlikely. Additionally, the similar extension between the LAT and the H.E.S.S. measurements disfavours further the PWN scenario, where usually a larger GeV nebula, due to cooling or/and energy-dependent diffusive transport, is observed (H. E. S. S. Collaboration 2019, 2012; Principe et al. 2020).

\subsection{Scenarios related to Westerlund 2 massive stars}

Electrons can also be efficiently accelerated in open clusters via shocks in, e.g. massive stars (Bednarek, Pabich \& Sobczak 2014), generating gamma-rays via IC or bremsstrahlung radiation. However, it is expected that the resulting gamma-rays should correlate with the region of high photon density. The gamma-ray emission extends up to $\sim 25_{d_{5 k p c}} \mathrm{pc}$, in contrast with the $\sim 4_{d_{5 k p c}} \mathrm{pc}$ core radius of the cluster, disfavouring a leptonic origin of the $\mathrm{GeV}$ and $\mathrm{TeV}$ emission observed. Likewise, the source size remains constant, within the errors, for different energy bands, which indicates a stable dependency of the cooling time with energy, contrary to what is expected in a leptonic scenario.

In contrast, a hadronic interpretation fits naturally the hard spectrum found from $\sim 200 \mathrm{MeV}$ up to a few tens of $\mathrm{GeV}$, where it connects smoothly with the H.E.S.S. spectrum, and continues up to a few TeVs before showing a drop of the flux. Next, we constrain the proton population that powers the gamma-ray source by modelling the $200-\mathrm{MeV}$ to $20-\mathrm{TeV}$ emission using the NAIMA package (version 0.8.4, Zabalza 2015). To calculate the SED, we used a distance of $5 \mathrm{kpc}$. The molecular content in the region has been deeply investigated by several authors (Dame 2007; Furukawa et al. 2014) using millimetre wave CO spectroscopy. Several massive molecular clouds were found within the surrounding of Westerlund 2. The total mass is estimated to be between $(1.7-7.5) \times 10^{5} M_{\odot}$. 
Table 2. Physical parameters of PSR J1023-5746 and its putative PWN. The pulsar rotation parameters $f$ and $\dot{f}$ are obtained from ATNF catalogue. The braking index $n$ and ejecta mass $M_{\mathrm{ej}}$ are fixed, following the results from Torres et al. (2014). The broken power-law spectral shape of electrons (defined with the indices $\alpha_{1,2}$ and breaking energy $\gamma_{\mathrm{b}}$ ) and magnetic field in the region $(B)$, which best represent the data, are listed in the third section of the table.

\begin{tabular}{lc}
\hline Pulsar and ejecta & \\
\hline$f(\mathrm{~Hz})$ & 8.97 \\
$\dot{f}\left(-10^{-12} \mathrm{~Hz} \mathrm{~s}^{-1}\right)$ & 30.88 \\
$\tau_{\mathrm{c}}(\mathrm{kyr})$ & 4.6 \\
$L\left(t_{\mathrm{age}}\right)(\mathrm{erg} / \mathrm{s})$ & $1.1 \times 10^{37}$ \\
$n$ & 2.509 \\
$D(\mathrm{kpc})$ & 5 \\
$M_{\mathrm{ej}}\left(M_{\odot}\right)$ & 10 \\
Environment & \\
$T_{\mathrm{FIR}}(\mathrm{K})$ & 30 \\
$w_{\mathrm{FIR}}\left(\mathrm{eV} / \mathrm{cm}^{3}\right)$ & 1 \\
$T_{*}(\mathrm{~K})$ & 2500 \\
$w_{*}\left(\mathrm{eV} / \mathrm{cm}^{3}\right)$ & 2 \\
$n_{\mathrm{H}}$ & 1.0 \\
& \\
Particles and field & \\
$\gamma_{\mathrm{b}}$ & $5 \times 10^{5}$ \\
$\alpha_{1}$ & 1.5 \\
$\alpha_{2}$ & 3.0 \\
$B\left(t_{\mathrm{age}}\right)(\mu \mathrm{G})$ & 7 \\
\hline
\end{tabular}

We used a particle distribution described by a particle index $s$ and an amplitude $N_{\mathrm{p}}$, up to an energy cutoff $\mathrm{E}_{\text {cutoff }}$. The corresponding gamma-ray emission due to pion decay radiation is calculated using the parametrization in Kafexhiu et al. (2014) implemented in NAIMA and compared to the experimental data. The best-fitting model for the joint Fermi-LAT and H.E.S.S. data corresponds to an exponential cutoff power-law proton spectrum with cutoff energy $\mathrm{E}_{\text {cutoff }}=93 \pm 8 \mathrm{TeV}$, and a particle index of $s=2.09 \pm 0.01$, referenced to $1 \mathrm{TeV}$, see Fig. 4). The fit to the data is done by means of a Log-Likelihood approach. For a distance of $5 \mathrm{kpc}$, the total energy in protons estimated above $1.22 \mathrm{GeV}$ (the threshold kinetic energy for pion production in pp interactions) is $W_{\mathrm{p}}=(1.3-5.9) \times$ $10^{48} \mathrm{erg}$ for densities of $n=(7.5-1.7) \times 10^{5} \mathrm{M}_{\odot} / V_{24 \mathrm{pc}}$, as derived by Furukawa et al. (2014) and Dame (2007), respectively. We estimated a lower limit for the cutoff energy on the proton spectrum of $\approx 37$ $\mathrm{TeV}$ (at 95 per cent CL) by comparing the maximum likelihoods of the data obtained for the exponential cutoff power law and power-law models with the likelihood-ratio test.

The total energy in protons (above $1.22 \mathrm{GeV}$ ) can be compared with the total mechanical power of the stellar winds in the Westerlund 2 cluster: $\mathrm{W}_{\text {tot }}=f L_{0} T_{0}$, which results in a modest acceleration efficiency of $f=10^{-4}\left(10^{-6} / 5 \times 10^{-3}\right)$, for the well-known age $\left(T_{0}=2 \times 10^{6} \mathrm{yr}\right)$, a distance of $5 \mathrm{kpc}(2 \mathrm{kpc} / 8 \mathrm{kpc})$, and the available energy budget in the form of kinetic energy of stellar winds $\left(L_{0}=\right.$ $2 \times 10^{38} \mathrm{erg} \mathrm{s}^{-1}$ ).

If we consider the total volume defined by the size of the $\mathrm{GeV}$ source ( $\sim 24$ pc, i.e. $V_{24 \mathrm{pc}}$ of volume for a spherical source), the energy density of protons in the region is $\omega_{\mathrm{p}}=(1.3-5.9) \times 10^{48} \mathrm{erg} / \mathrm{V}_{24 \mathrm{pc}} \simeq$ $(0.5-2.2) \mathrm{eV} / \mathrm{cm}^{3}$ (or $0.1-5.6 \mathrm{eV} / \mathrm{cm}^{3}$ if considering the uncertainty due to the distance to the source), which is comparable to the density of protons found in others massive clusters (Aharonian et al. 2019). The maximum energy of this proton population is constrained by the best fit, described above, to be $\sim 90 \mathrm{TeV}$, with a lower limit

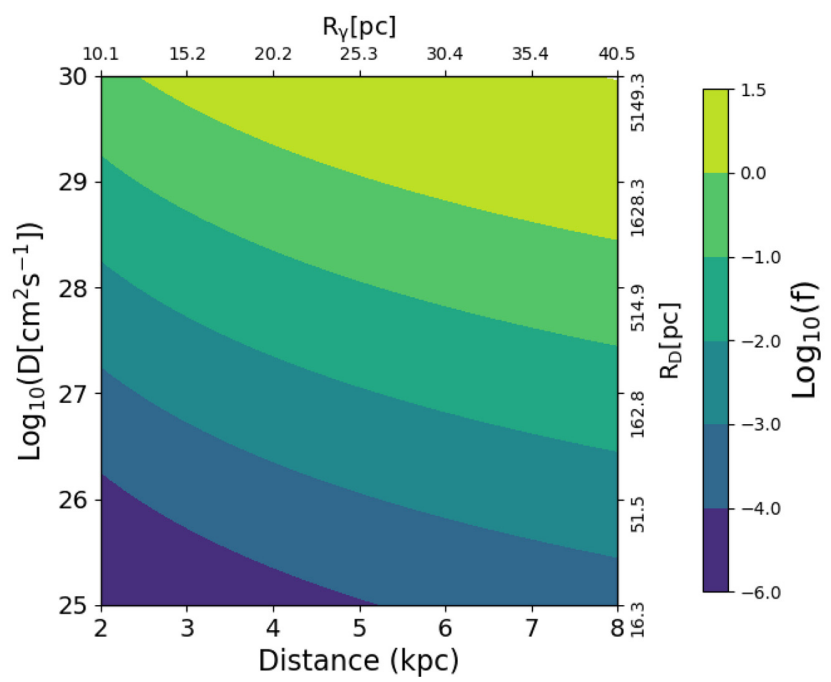

Figure 5. Acceleration efficiency $(f)$ obtained with respect to the distance and diffusion coefficient $(D)$ assumed (for a fiducial target mass in molecular clouds of $4.5 \times 10^{5} M_{\odot}$ ). The upper and right axes correspond to the estimated gamma-ray source and CR's diffusion radius, respectively.

of $37 \mathrm{TeV}$ on the energy cutoff. It would be possible, however, that a significant part of these CRs has already escaped from the gammaray emission region, where the molecular content is enhanced, and therefore also the gamma-ray radiation. Under the assumption of a continuous injection of protons and spherical expansion (Aharonian \& Atoyan 1996), the relation between the observed energy emitted by protons $\left(W_{\mathrm{em}}\right)$ and the total energy (in protons) available $\left(W_{\mathrm{tot}}\right)$ is:

$W_{\mathrm{em}} / W_{\mathrm{tot}}=\left(R_{\gamma} / R_{\mathrm{D}}\right)^{2}$,

where $R_{\gamma}$ and $R_{\mathrm{D}}$ are the gamma-ray source and diffusion radii, respectively. Then, using a diffusion coefficient as in the ISM, i.e. $D \sim 10^{28} \mathrm{~cm}^{2} \mathrm{~s}^{-1}$ and $R_{\mathrm{D}}=2 \sqrt{T_{0} D}=515 \mathrm{pc}$, we obtain that the total energy released in the form of CRs could reach $W_{\text {tot }} \sim 5 \times$ $10^{50} \mathrm{erg}$ (for a distance of $5 \mathrm{kpc}$ ), which is still a few percent of the total available energy in the kinetic winds $\left(L_{0} T_{0} \sim 10^{52} \mathrm{erg}\right)$. Note that this number is affected by the uncertainties in the distance: for instance, the efficiency obtained is larger than 10 percent for a distance of $8 \mathrm{kpc}$ with the quoted diffusion coefficient of the ISM. Likewise, formally, the value of the diffusion coefficient could be larger and, correspondingly, the CR halo could reach up to $5 \mathrm{kpc}$ (for $\left.D \sim 10^{30} \mathrm{~cm}^{2} \mathrm{~s}^{-1}\right)$. These uncertainties have an effect on the total efficiency, reaching in some extreme cases an unrealistically large fraction of the total energy to be transferred to CRs.

If the region is, instead, affected by large turbulence, expected in the surrounding of an accelerator, the CR's diffusion could be much slower (Malkov et al. 2013; Schroer et al. 2020). If the size observed at $\mathrm{GeV}$ and $\mathrm{TeV}$ energies $\left(R_{\gamma} \sim 24(d / 5 \mathrm{kpc})\right.$ parsecs $)$ reflects the propagation depth of CRs, the diffusion coefficient would be much lower than in the ISM $D \sim 3 \times 10^{25} \mathrm{~cm}^{2} \mathrm{~s}^{-1}$ [or $(0.4-6) \times 10^{25} \mathrm{~cm}^{2} \mathrm{~s}^{-1}$ for 2 and $8 \mathrm{kpc}$, respectively], which is in tension with the value of the diffusion coefficient at these energies in the Bohm regime (Aharonian et al. 2019). That points to a certain CR halo around Westerlund 2, beyond the size traced by LAT and H.E.S.S., which could, in principle, extend up to a few tens of parsecs. The discussion above is roughly summarized in Fig. 5. More precise estimations of the distance to the cluster, foreseen with Gaia DR3 (Zeidler et al. 2018) (and therefore of the real gamma-ray size), would provide 
constraining limits on the diffusion of CRs around the source for a range of acceleration efficiency $f$.

Another possibility to explain the gamma-ray emission involves energetic supernova remnant (SNR) explosions within the cluster, injecting CRs in the surrounding (Townsley et al. 2019). The lack of shell-like structure and the high efficiency required in the case of diffusive CRs (50 per cent $E_{\mathrm{SN}}$, for a standard $E_{\mathrm{SN}}=10^{51} \mathrm{erg}$ ) render this hypothesis less attractive, at least for a single SNR event, than the one that attributes the origin of CRs to the stellar winds.

\section{CONCLUSIONS}

The reanalysis of the large LAT data set presented here results in a clear identification of the extended source FGES J1023.3-5747 with the TeV source HESS J1023-575. The matching spectral and morphological agreement, with no signs of cooling features in the size of the source, points to a common origin of the radiation. The combination of the two results obtained, that is, the extended source beyond the cluster size and in a good agreement with the $\mathrm{TeV}$ radiation, and the hard spectrum that continues towards low energies, constitutes evidence of the hadronic nature of the gamma-ray emission detected using Fermi-LAT and H.E.S.S. data. Here, we evaluated the different scenarios proposed to explain HESS J1023-575, in light of the new information provided by the LAT spectrum and concluded that the gamma-ray source is compatible with being of hadronic origin, and related to the Westerlund 2 stellar cluster, rather than to leptonic emission from either the PWN associated with PSR J1023-5746 or the cluster itself. However, the PWN scenario, even if unlikely, cannot be conclusively ruled out when considering the uncertainties in the data points. Moreover, the reasonable uncertainties of both models as well as the possible existence of a multicomponent photon energy density introduce further ambiguity.

The results presented, pointing to the Westerlund 2 cluster as a hadronic accelerator with a hard $\sim 2$ spectral index, indirectly support the hypothesis of stellar clusters as significant contributors to the GCR sea. In particular, these CRs steaming from Westerlund 2 might also extend to a very large halo ( $\sim 200$-pc radius) around the cluster, as proposed in Yang et al. (2018). The total energy in protons we derive $\left(\sim 5 \times 10^{50} \mathrm{erg}\right)$ can easily account for the total luminosity observed, requiring an acceleration efficiency in the cluster of $f=$ 0.04 , which is still moderate for acceleration theories in wind shocks. The spectrum at $\mathrm{TeV}$ energies seems to change the hard 2 index trend found in the LAT data, softening towards higher energies. This spectral shape would imply a low-energy cutoff, disfavouring Westerlund 2 as a PeVatron accelerator. Deeper observations with H.E.S.S. or with sensitive $\mathrm{TeV}$ instruments in the South such as CTA (Cherenkov Telescope Array Consortium et al. 2019) in the future should provide a definitive answer to the PeVatron nature of HESS J1023-575.

\section{ACKNOWLEDGEMENTS}

This research was supported by the Alexander von Humboldt Foundation (EdOW). EM and D FT acknowledge the support of the grants AYA2017-92402- EXP, PGC2018-095512-B-I00 (Ministerio de Economía y Competitividad), and SGR2017-1383 (Generalitat de Catalunya). We acknowledge the support of the PHAROS COST Action (CA16214). We made use of R Project for Statistical Computing (R Core Team 2013). The Fermi-LAT Collaboration acknowledges generous ongoing support from a number of agencies and institutes that have supported both the development and the operation of the LAT as well as scientific data analysis. These include the National
Aeronautics and Space Administration and the Department of Energy in the United States, the Commissariat a' l'Energie Atomique and the Centre National de la Recherche Scientifique/Institut National de Physique Nucléaire et de Physique des Particules in France, the Agenzia Spaziale Italiana and the Istituto Nazionale di Fisica Nucleare in Italy, the Ministry of Education, Culture, Sports, Science and Technology (MEXT), High Energy Accelerator Research Organization (KEK) and Japan Aerospace Exploration Agency (JAXA) in Japan, the K. A. Wallenberg Foundation, the Swedish Research Council, and the Swedish National Space Board in Sweden. This work performed in part under DOE Contract DE-AC02-76SF00515.

\section{DATA AVAILABILITY}

The Fermi-LAT data underlying this article are available at: http s://fermi.gsfc.nasa.gov/ssc/data/access/lat/. The GLIMPSE survey archival data underlying Fig. 3 are available at: https://irsa.ipac.calt ech.edu/data/SPITZER/GLIMPSE/.

\section{REFERENCES}

Abdo A. A. et al., 2013, ApJS, 208, 17

Abdollahi S. et al., 2020, ApJS, 247, 33

Abramowski A. et al., 2012, A\&A, 537, A114

Acero F. et al., 2013, Astrophys. J., 773, 77

Ackermann M. et al., 2011a, Science, 334, 1103

Ackermann M. et al., 2011b, ApJ, 726, 35

Ackermann M. et al., 2017a, ApJ, 843, 139

Ackermann M. et al., 2017b, ApJ, 843, 139

Aharonian F. et al., 2007, A\&A, 467, 1075

Aharonian F., Yang R., de Oña Wilhelmi E., 2019, Nature Astron., 3, 561

Aharonian F. A., Atoyan A. M., 1996, A\&A, 309, 917

Ajello M. et al., 2011, ApJ, 744, 80

Ascenso J., Alves J., Beletsky Y., Lago M. T. V. T., 2007, A\&A, 466, 137

Atwood W. et al., 2013, 2012 Fermi Symposium proceedings, preprint (arXiv:1303.3514)

Atwood W. B. et al., 2009, ApJ, 697, 1071

Ballet J., Burnett T. H., Digel S. W., Lott B., 2020, preprint (arXiv:2005.11208)

Bednarek W., Pabich J., Sobczak T., 2014, Phys. Rev. D, 90, 103008

Binns W. R. et al., 2005, ApJ, 634, 351

Blumenthal G. R., Gould R. J., 1970, Rev. Mod. Phys., 42, 237

Bonanos A. Z. et al., 2004, ApJ, 611, L33

Bruel P., Burnett T. H., Digel S. W., Johannesson G., Omodei N., Wood M., 2018, Fermi-LAT improved Pass 8 event selection (8th International Fermi Symposium) preprint (arXiv:1810.11394)

Bykov A. M., Marcowith A., Amato E., Kalyashova M. E., Kruijssen J. M. D., Waxman E., 2020, Space Sci. Rev., 216, 42

Carraro G., Munari U., 2004, MNRAS, 347, 625

Cesarsky C. J., Montmerle T., 1983, Space Sci. Rev., 36, 173

Cherenkov Telescope Array Consortium et al., 2019, Science with the Cherenkov Telescope Array, World Scientific Publishing Co. Pte. Ltd.

Dame T. M., 2007, ApJ, 665, L163

Drew J. E., Herrero A., Mohr-Smith M., Monguió M., Wright N. J., Kupfer T., Napiwotzki R., 2018, MNRAS, 480, 2109

Fujita Y., Hayashida K., Takahashi H., Takahara F., 2009, PASJ, 61, 1229

Furukawa N. et al., 2014, ApJ, 781, 70

Furukawa N., Dawson J. R., Ohama A., Kawamura A., Mizuno N., Onishi T., Fukui Y., 2009, ApJ, 696, L115

Gabici S., Evoli C., Gaggero D., Lipari P., Mertsch P., Orlando E., Strong A., Vittino A., 2019, Int. J. Mod. Phys. D, 28, 1930022

Guarnieri M. D., Lattanzi M. G., Massone G., Munari U., Moneti A., 1995, The Formation of the Milky Way, IAA-IAC-University of Pisa Workshop. p. 183

H. E. S. S. Collaboration 2011, A\&A, 525, A46 
H. E. S. S. Collaboration 2012, A\&A, 548, A46

H. E. S. S. Collaboration 2015, Science, 347, 406

H. E. S. S. Collaboration 2018, A\&A, 612, A2

H. E. S. S. Collaboration 2019, A\&A, 621, A116

Hahn J., 2015, in 34th International Cosmic Ray Conference (ICRC2015). Sissa Medialab srl Partita, The Hague, The Netherlands, p. 917

Hobbs G. B., Edwards R. T., Manchester R. N., 2006, MNRAS, 369, 655

Kafexhiu E., Aharonian F., Taylor A. M., Vila G. S., 2014, Phys. Rev. D, 90, 123014

Kerr M., Ray P. S., Johnston S., Shannon R. M., Camilo F., 2015, ApJ, 814, 128

Lande J. et al., 2012, ApJ, 756, 5

Li J., Torres D. F., Lin T. T., Grondin M.-H., Kerr M., Lemoine-Goumard M., de Oña Wilhelmi E., 2018, ApJ, 858, 84

Malkov M. A., Diamond P. H., Sagdeev R. Z., Aharonian F. A., Moskalenko I. V., 2013, ApJ, 768, 73

Manolakou K., Horns D., Kirk J. G., 2007, A\&A, 474, 689

Mattox J. R. et al., 1996, ApJ, 461, 396

Moffat A. F. J., Shara M. M., Potter M., 1991, AJ, 102, 642

Piatti A. E., Bica E., Claria J. J., 1998, A\&A, 127 S423

Principe G., Mitchell A. M. W., Caroff S., Hinton J. A., Parsons R. D., Funk S., 2020, A\&A, 640, A76

R Core Team, 2013, R: A Language and Environment for Statistical Computing. R Foundation for Statistical Computing, Vienna, Austria, Available at: http://www.R-project.org/

Rauw G. et al., 2004, A\&A, 420, L9

Rauw G. et al., 2005, A\&A, 432, 985

Rauw G., Manfroid J., Gosset E., Nazé Y., Sana H., De Becker M., Foellmi C., Moffat A. F. J., 2007, A\&A, 463, 981

Saha L., Domínguez A., Tibaldo L., Marchesi S., Ajello M., LemoineGoumard M., López M., 2020, ApJ, 897, 131

Salpeter E. E., 1955, ApJ, 121, 161

Saz Parkinson P. M. et al., 2010, ApJ, 725, 571

Scargle J. D., Norris J. P., Jackson B., Chiang J., 2013, ApJ, 764, 167

Schroer B., Pezzi O., Caprioli D., Haggerty C., Blasi P., 2020, preprint (arXiv:2011.02238)

Sun X.-N., Yang R.-Z., Wang X.-Y., 2020, MNRAS, 494, 3405

Torres D. F., Cillis A., Martín J., de Oña Wilhelmi E., 2014, J. High Energy Astrophy., 1, 31

Townsley L. K., Broos P. S., Garmire G. P., Povich M. S., 2019, ApJS, 244, 28

Tsujimoto M. et al., 2007, ApJ, 665, 719

Uzpen B. et al., 2005, ApJ, 629, 512

Vargas Álvarez C. A., Kobulnicky H. A., Bradley D. R., Kannappan S. J., Norris M. A., Cool R. J., Miller B. P., 2013, AJ, 145, 125
Whiteoak J. B. Z., Uchida K. I., 1997, A\&A, 317, 563

Whitney B. A. et al., 2004, ApJS, 154, 315

Wood M., Caputo R., Charles E., Di Mauro M., Magill J., Perkins J. S., FermiLAT Collaboration, 2017, in 35th International Cosmic Ray Conference (ICRC2017). p. 824, preprint (arXiv:1707.09551)

Yang R.-Z., de Oña Wilhelmi E., Aharonian F., 2018, A\&A, 611, A77

Yang R.-Z., Wang Y., 2020, A\&A, 640, A60

Zabalza V., 2015, Proc. of International Cosmic Ray Conference 2015. Busan, Korea, p. 922

Zeidler P. et al., 2018, AJ, 156, 211

\section{APPENDIX A: ANALYSIS DETAILS}

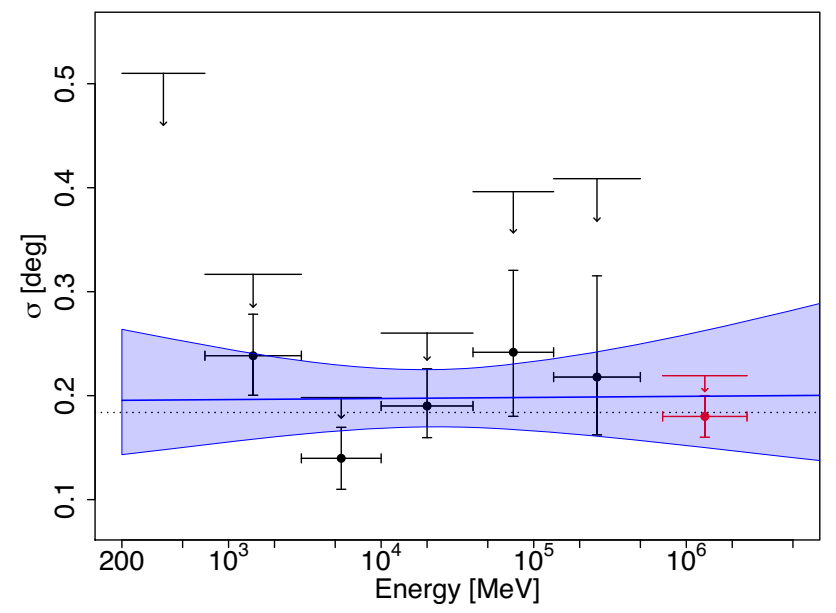

Figure A1. Extension of FGES J1023.3-5747 (i.e. intrinsic width for a Gaussian fit) measured for different energy bins (in black points for LAT data, with only statistical errors). The red point corresponds to the intrinsic extension measured by H.E.S.S. from $0.7 \mathrm{TeV}$ to $2.5 \mathrm{TeV}$ of energy (H. E. S. S. Collaboration 2011). The best-fitting power-law model (for the joint LAT and H.E.S.S. data) is plotted in blue line with the $1 \sigma$ region noted (the blue shaded area), together with the weighted mean size (black dashed line). The arrows correspond to an upper limit for the extension (i.e. $\sigma$, at 95 per cent $\mathrm{CL}$ ), except for the one in the first energy bin corresponding with the upper limit for the 95 per cent containment radius $\left(r_{95}\right)$. 
Table A1. Best-fitting position (in equatorial and Galactic coordinates) for PSR J1023-5746 and FGES J1023.3-5747 (in degrees, with only statistical errors).

\begin{tabular}{lcccc}
\hline Parameter & PSR J1023-5746 (on-peak) & PSR J1023-5746 (Bridge) & FGES J1023.3-5747 (Off-peak) & FGES J1023.3-5747 (E > 10 GeV) \\
\hline RA & $155.772 \pm 0.005$ & $155.79 \pm 0.01$ & $155.93 \pm 0.03$ & $155.93 \pm 0.02$ \\
DEC & $-57.764 \pm 0.005$ & $-57.77 \pm 0.01$ & $-57.79 \pm 0.03$ & $-57.76 \pm 0.02$ \\
$l$ & $284.168 \pm 0.005$ & $284.18 \pm 0.01$ & $284.25 \pm 0.02$ & $-0.38 \pm 0.03$ \\
$b$ & $-0.401 \pm 0.005$ & $-0.40 \pm 0.01$ & & $-0.35 \pm 0.02$ \\
& & & & \\
Parameter & FGES J1023.3-5747 (on-peak) & FGES J1023.3-5747 (Bridge) & \\
RA & $155.98 \pm 0.04$ & $155.83 \pm 0.03$ & \\
DEC & $-57.77 \pm 0.03$ & $-57.81 \pm 0.03$ & \\
$l$ & $284.27 \pm 0.05$ & $284.22 \pm 0.03$ & \\
$b$ & $-0.35 \pm 0.03$ & $-0.43 \pm 0.03$ & \\
\hline
\end{tabular}

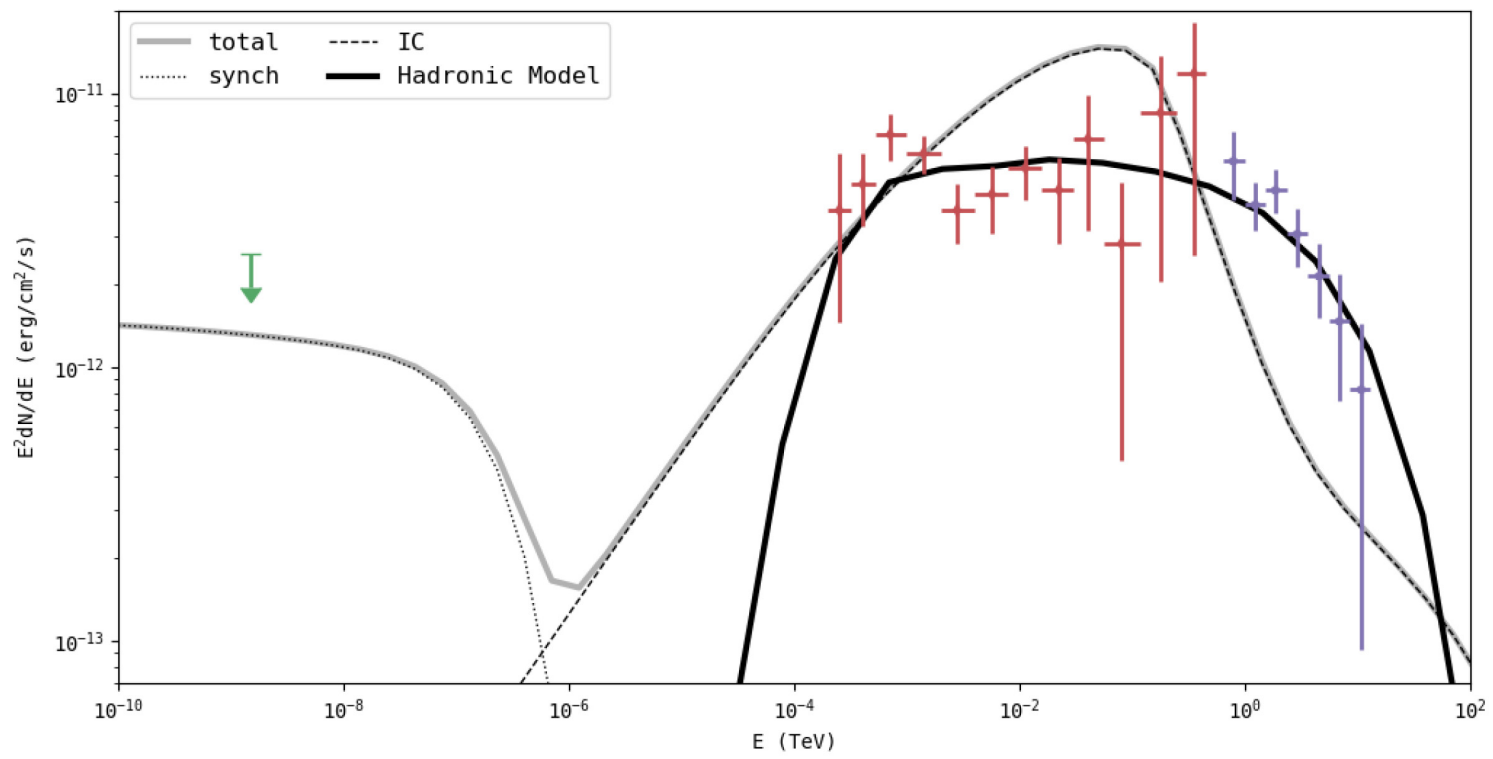

Figure A2. The same as Fig. 4, but the best-fitting model for the PWN hypothesis depicted (dashed line) corresponds to a maximum stellar photon field of $w_{*}$ $=500 \mathrm{eV} / \mathrm{cm}^{3}$ at a temperature of $T=3 \times 10^{4} \mathrm{~K}$ as in Manolakou et al. (2007).

This paper has been typeset from a $\mathrm{T}_{\mathrm{E}} \mathrm{X} / \mathrm{L} \mathrm{T} \mathrm{E} \mathrm{X}$ file prepared by the author. 